\title{
Caracterização de Ambientes de Produção para Amendoim na Região Alta Paulista
}

Submetido - 27 jul. 2020

Aprovado - 10 set. 2020

Publicado - 14 out. 2020

http://dx.doi.org/10.17648/sas.v1i2.41

\begin{abstract}
Dr., Pesquisador Científico - IAC, Centro de Cana, Ribeirão Preto, SP, e-mail: denizart@iac.sp.gov.br.
Téc. Agrícola - Representante Técnico Comercial, TIMAC Agro, Tupã, SP.

Adriano Frisneda (iD
\end{abstract}

Denizart Bolonhezi (i)

Olavo Betiol (D)

Eng..$^{\circ}$ Agrônomo, Mestrando em Agronomia - Departamento de Solos, FCAV/Unesp, Jaboticabal, SP e e-mail: olavobetiol96@gmail.com.

Élcio Ríos Perez Leal(D)

Eng. ${ }^{\circ}$ Agrônomo, Mestrando em Agronomia - Departamento de Solos, FCAV/Unesp, Jaboticabal,SP e e-mail: elciorpleal@gmail.com.

Eng..$^{\circ}$ Agrônomo - AGROTEKENE, Tupã, SP.

José Paulo de Oliveira Lollato (iD

Angélica Prelá Pantano iD

Dra. Pesquisadora Científica - IAC, Centro de Ecofisiologia e Biofísica, Campinas, SP, e-mail: angelica@iac.sp.gov.br.

\section{RESUMO}

Não há informação sistematizada para o amendoim sobre caracterização de ambientes de produção, assim como há dúvidas sobre os principais fatores que determinam altas produtividades. Com os objetivo de identificar quais fatores determinaram altas produtividades $e$ iniciar a construção do conceito de ambientes de produção para o amendoim, foram monitoradas as colheitas em 45 áreas comerciais nas safras 2017/2018, 2018/2019 e 2019/2020 na região de Tupã/SP. Foi proposta metodologia de avaliação da produtividade (entre 1,8 e 2,42 ha) e levantadas informações de solo e microclima em cada área. Os resultados permitiram identificar quatro estratos de produtividade (> 6200, 6200-5175, 5175-4125 e <4125 kg ha-1), com frequência variando com a ocorrência de deficiência hídrica que reduziu em $23 \%$ a produtividade na safra 2018/2019. Houve correlação positiva entre produtividade e chuva acumulada $(r=0,52)$, teores de matéria orgânica $(r=0,52)$ e negativa com 0 atraso da semeadura $(r=-0,37)$. Conclui-se que o conceito de ambiente de produção para amendoim deve envolver também o efeito de outros fatores, tais como; histórico de cultivo e conteúdo de carbono orgânico.

Palavras-chave: Arachis hypogaea L.; Pedologia; Clima; Produtividade.

\section{Characterization of Production Environment for Peanut in Alta Paulista Region}

\begin{abstract}
There is no organized information for peanut about the characterization of environment production, as well as there are many doubts in terms of the factors that influencing the high yield. In order to identify which factors determining high yield and to start developing of the production environment for peanut, it was evaluated and harvested 45 commercial field during
\end{abstract}


the growing season 2017/2018, 2018/2019 and 2019/2020 in Tupa city, Sao Paulo State. A methodology was developed to evaluate the pod yield (area from 1,8 to 2,42 hectare), as well as information of soil and weather were collected. The results could identify four levels of productivity ( $>6200,6200-5175,5175-4125$ and $<4125 \mathrm{~kg} \mathrm{ha}^{-1}$ ) has changed the frequency according to the occurrence of water deficiency, which could diminish the pod yield around $23 \%$ in the growing season 2018/19. There was positive correlation between pod yield and rainfall $(r=0,52)$, with soil organic matter $(r=0,52)$, and negative correlation with late sowing date $(r=-0,37)$. It could be concluded that the concept of production environment for peanut is dependent of other factors, such as; historic of field in terms of crop cultivated before and the soil organic matter.

Keywords: Arachis hypogaea L.; Pedology; Rainfall; Yield.

\title{
Caracterización del Ambiente de la Producción para Maní en la Zona Manisera Alta Paulista
}

\author{
RESUMEN
}

\begin{abstract}
No hay información organizada a respecto de la caracterización del ambiente de producción del maní, así como hay muchas dudas cuanto a los factores que puden definir altos rendimentos. Para identificar qué factores determinaron la alta productividad y comenzar a construir el concepto de ambiente de producción para el maní, 45 fincas comerciales fueron cosechadas en las temporadas 2017/18, 2018/19 y 2019/20 en la región de Tupã / SP. Fue hecha una metodología para evaluar la productividad (entre 1.8 y 2.42 ha) y se recopiló información de suelo y clima en cada finca. Los resultados permitieron identificar cuatro estratos de productividad (> 6200, 6200-5175, 5175-4125 e < $4125 \mathrm{~kg} \mathrm{ha}^{-1}$ ) variando con la ocurrencia de deficiencia de agua que he disminuido la productividad en un $23 \%$. Hubo una correlación positiva entre la productividad y la Iluvia acumulada $(r=0.52)$, el contenido de carbón orgánico ( $r=0.52)$ y una correlación negativa con el retraso de la siembra $(r=-0.37)$. Se concluye que el concepto de ambiente de producción para el maní, también debe involucrar el efecto de otros factores, tales como; cultivo anterior y contenido carbón orgánico.
\end{abstract}

Palabras clave: Arachis hypogaea L.; Pedologia; lluvia; Productividad.

\section{Introdução}

A cadeia de produção do amendoim está concentrada no Estado de São Paulo em dois principais polos, nas regiões de Jaboticabal/SP ("Alta Mogiana") e Tupã/SP (“Alta Paulista"). Nas duas regiões a cultura passou por drásticas transformações tecnológicas, sendo que nos últimos 10 anos nas terras paulistas; a área, a produção e a produtividade cresceram 46, 67 e 13\%, respectivamente (CONAB, 2020).

A diferença entre o potencial produtivo e o real obtido é conhecido na literatura como yield gap e tem sido alvo de diversas pesquisas com várias culturas, no sentido de explicar as causas que penalizam a produtividade. De acordo com Sentelhas et al. (2015), a deficiência hídrica e o manejo da cultura são responsáveis por $74 \%$ e $26 \%$ do yield gap da soja 
no Brasil. Para a cultura do amendoim, Sonawane et al. (2016) concluíram para condições da Índia, que produtores de bom nível tecnológico reduzem em média $30 \%$ o yield gap.

Mas compreender as características do ambiente de produção também é fundamental para diminuir essa discrepância. A exemplo da canade-açúcar, que construiu com muita propriedade as matrizes de ambientes de produção, que tem como base a pedologia e as informações do clima.

A proposta do IAC para cana-de-açúcar é dinâmica e apresenta pelo menos 10 ambientes de produção (PRADO; 2011) e é imprescindível para posicionamento das variedades quanto à época de plantio e à época de colheita (LANDELL el al., 2003). Contudo, não há conhecimento sistematizado, que permita estabelecer relações entre as características do ambiente e produtividade do amendoim.

Nesse sentido, foram realizadas nas últimas três safras (2017/18, 2018/19 e 2019/2020) na região produtora de Tupã/SP, 45 levantamentos da produtividade em nível de produtor, com os objetivos de; identificar em nível de campo quais as faixas de rendimentos e quais fatores determinaram esses tetos produtivos, de forma a iniciar a construção do conceito de ambientes de produção para o amendoim

\section{Material e métodos}

Os produtores participantes foram os cadastrados no CRAT (Concurso Regional de Amendoim da TIMAC), organizado pela TIMAC Agro na região de abrangência do polo produtivo de Tupã/SP, nas safras 2017/2018, 2018/2019 e 2019/2020.

Foi adaptada a metodologia utilizada no prêmio CESB (Comitê Estratégico Soja Brasil), que consiste em colher 5 ha, porém considerou-se módulos de colheita até 2,42 hectare, escolhidos em um talhão de 10 ha.

$\mathrm{Na}$ safra 2017/18, 2018/19 e 2019/20 foram acompanhadas as colheitas em 11, 15 e 19 produtores, respectivamente. Criou-se um banco de 
dados de cada área nos três anos, que foram numeradas de 1 a 45, incluindo informações quanto ao histórico do talhão (cultura anterior), cultivar, semeadora (dupla ou simples), localização geográfica e altitude do local, análise química da fertilidade do solo, data de semeadura e arranquio (Tabelas 1, 2 e 3).

Por ocasião do recolhimento das leiras, foram retiradas amostras de terra estratificadas nas camadas $0-10,10-20,20-30,30-40,40-60,60-80$ e 80-100 cm de profundidade, com finalidade de avaliação da textura e posterior classificação pedológica do solo. O recolhimento foi efetuado com equipamento do produtor e a produtividade foi aferida com uso de balança digital com capacidade para até $20 \mathrm{mil} \mathrm{kg}$.

A área destinada para colheita apresentou layout variável, devido as diferentes condições das glebas e existência de terraços, mas oscilou entre 1,8 e 2,42 hectares, medidos com receptores de GNSS (Global Navigation Satellite System). Após pesagens dos transbordos, foram retiradas amostras do amendoim colhidos (entre 15 e $25 \mathrm{~kg}$ ) para quantificação do percentual de impurezas (realizada em laboratório da APTA) e do teor de água nos grãos (descascamento no mesmo dia).

Para a determinação do teor de água nos grãos foi utilizado equipamento digital de marca Gehaka ${ }^{\circledR}$. A produtividade final em kg ha-1 foi corrigida para umidade de $8 \%$ e descontados os valores das impurezas.

Os dados das safras 2017/2018, 2018/2019 e 2019/2020 foram analisados de acordo com estatística descritiva e análise de correlação simples entre a produtividade de vagens com as variáveis; chuva acumulada, teores de matéria orgânica, teores de fósforo, altitude, percentual de argila e data de semeadura. 
Tabela 1. Informações das 11 áreas de amendoim colhidas na safra 2017/18, na região Alta Paulista, utilizando participantes do CRAT da TIMAC Agro.

\begin{tabular}{|c|c|c|c|c|c|c|c|}
\hline \multirow{2}{*}{ N. ${ }^{\circ}$} & \multirow{2}{*}{ Cidade } & \multirow{2}{*}{ Alt. (m) } & \multirow{2}{*}{ Histórico } & \multirow{2}{*}{ Linha } & \multirow{2}{*}{ Cultivar } & \multicolumn{2}{|c|}{ Data } \\
\hline & & & & & & Sem.* & Colheita \\
\hline 01 & Rinópolis & 441 & Pastagem & Dupla & IAC-OL3 & $04 / 10$ & $22 / 02$ \\
\hline 02 & Herculândia & 446 & Cana & Simples & IAC-OL3 & $06 / 10$ & $26 / 02$ \\
\hline 03 & Queiróz & 448 & Pastagem & Dupla & IAC-OL3 & $06 / 10$ & $01 / 03$ \\
\hline 04 & Varpa & 403 & Milho & Simples & $\begin{array}{c}\text { Granolêic } \\
0\end{array}$ & $26 / 10$ & $12 / 03$ \\
\hline 05 & P. Paulista & 522 & Cana & Dupla & $\begin{array}{c}\text { Granolêic } \\
0\end{array}$ & $16 / 11$ & $20 / 03$ \\
\hline 06 & Herculândia & 502 & Cana & Simples & IAC-OL3 & $20 / 11$ & $27 / 03$ \\
\hline 07 & Getulina & 425 & Cana & Simples & $\begin{array}{c}\text { Granolêic } \\
0\end{array}$ & $16 / 11$ & $05 / 04$ \\
\hline 08 & Planalto & 425 & Cana & Simples & IAC-503 & $11 / 11$ & $12 / 03$ \\
\hline 09 & Nantes & 476 & $\begin{array}{c}\text { Pasto/4xA } \\
\mathrm{m} .\end{array}$ & Simples & IAC-505 & $20 / 11$ & $14 / 04$ \\
\hline 10 & Getulina & 448 & $\begin{array}{c}\text { Cana/Am./ } \\
\mathrm{Mi}\end{array}$ & Dupla & $\begin{array}{c}\text { Granolêic } \\
0\end{array}$ & $27 / 10$ & $14 / 04$ \\
\hline 11 & Getulina & 532 & $\begin{array}{c}\text { Cana/2x } \\
\text { Am. }\end{array}$ & Dupla & G. Green & $06 / 12$ & $15 / 04$ \\
\hline
\end{tabular}

Tabela 2. Informações das 15 áreas de amendoim colhidas na safra 2018/19, na região Alta Paulista, utilizando participantes do CRAT da TIMAC Agro. ("Pasto/Amendoim/Milheto).

\begin{tabular}{|c|c|c|c|c|c|c|c|}
\hline \multirow{2}{*}{ N. ${ }^{\circ}$} & \multirow{2}{*}{ Cidade } & \multirow{2}{*}{$\begin{array}{l}\text { Alt. } \\
(\mathrm{m})\end{array}$} & \multirow{2}{*}{ Histórico } & \multirow{2}{*}{ Linha } & \multirow{2}{*}{ Cultivar } & \multicolumn{2}{|c|}{ Data } \\
\hline & & & & & & Sem. & Colh. \\
\hline 12 & Rinópolis & 417 & Pastagem & Dupla & Granolêico & $28 / 09$ & $08 / 02$ \\
\hline 13 & Tupã & 513 & Pastagem & Dupla & Granolêico & $06 / 10$ & $06 / 02$ \\
\hline 14 & Queiroz & 430 & $\mathrm{~Pa} / \mathrm{Am} / \mathrm{Mi}$ & Simples & IAC-OL3 & $21 / 09$ & 09/02 \\
\hline 15 & Nantes & 454 & $\begin{array}{c}\text { Milho/Ave } \\
\text { ia }\end{array}$ & Simples & IAC-505 & $06 / 11$ & $01 / 04$ \\
\hline 16 & Getulina & 480 & Cana & Dupla & Granolêico & $31 / 10$ & $17 / 03$ \\
\hline 17 & Quatá & 490 & Pastagem & Dupla & IAC-OL3 & $15 / 10$ & $07 / 03$ \\
\hline 18 & Quatá & 442 & Am/Mand. & Simples & IAC-OL3 & $22 / 09$ & $22 / 02$ \\
\hline 19 & Queiroz & 413 & Cana & Simples & Granolêico & $30 / 10$ & $13 / 03$ \\
\hline 20 & $\begin{array}{l}\text { Herculând } \\
\text { ia }\end{array}$ & 506 & Cana & Simples & IAC-OL3 & $18 / 10$ & $20 / 03$ \\
\hline 21 & Getulina & 442 & Cana & Dupla & IAC-OL3 & $18 / 10$ & $08 / 03$ \\
\hline
\end{tabular}




\begin{tabular}{cccccccc}
22 & lacri & 450 & Cana & Simples & Granolêico & $5 / 12$ & $17 / 04$ \\
23 & Quintana & 464 & Cana & Dupla & IAC-OL3 & $20 / 09$ & $01 / 03$ \\
24 & Luiziânia & 412 & Cana & Dupla & IAC-OL3 & $31 / 11$ & $13 / 03$ \\
25 & Tupã & 480 & Cana & Dupla & IAC-OL3 & $7 / 11$ & $26 / 03$ \\
\hline 26 & Queiroz & 413 & Cana & Simples & Granolêico & $30 / 10$ & $13 / 03$ \\
\hline
\end{tabular}

Tabela 3. Informações das 19 áreas de amendoim colhidas na safra 2019/20, na região Alta Paulista, utilizando os participantes do CRAT da TIMAC Agro. ( ${ }^{*}$ Pasto/Amendoim/Milheto/Amendoim)

\begin{tabular}{|c|c|c|c|c|c|c|c|}
\hline \multirow{2}{*}{ N. ${ }^{\circ}$} & \multirow{2}{*}{ Cidade } & \multirow{2}{*}{$\begin{array}{l}\text { Alt. } \\
\text { (m) }\end{array}$} & \multirow{2}{*}{ Histórico } & \multirow{2}{*}{ Linha } & \multirow{2}{*}{ Cultivar } & \multicolumn{2}{|c|}{ Data } \\
\hline & & & & & & Sem. & Colheita \\
\hline 27 & Arco Íris & 444 & Pastagem & Dupla & IAC-OL3 & $21 / 09$ & $14 / 02$ \\
\hline 28 & Arco Íris & 453 & Pastagem & Simples & IAC-OL3 & $28 / 09$ & $14 / 02$ \\
\hline 29 & Luiziânia & 446 & Cana & Simples & IAC-OL3 & 22/09 & $29 / 02$ \\
\hline 30 & Universo & 477 & $\begin{array}{c}\text { Cana/2x } \\
\text { Amendoim }\end{array}$ & Simples & $\begin{array}{c}\text { Granolêic } \\
0\end{array}$ & $23 / 10$ & 03/03 \\
\hline 31 & Rinópolis & 480 & Pastagem & Dupla & $\begin{array}{c}\text { Granolêic } \\
0\end{array}$ & $02 / 10$ & 03/03 \\
\hline 32 & Quatá & 539 & Pastagem & Dupla & IAC-OL3 & $15 / 10$ & 05/03 \\
\hline 33 & Taçiba & 466 & Pastagem & Dupla & IAC-OL3 & $22 / 10$ & 05/03 \\
\hline 34 & Tupã & 438 & Pa./Am./Mi/Am. & Dupla & IAC-505 & $27 / 10$ & 06/03 \\
\hline 35 & Guaimbê & 435 & Cana & Dupla & IAC-OL3 & $28 / 10$ & 09/03 \\
\hline 36 & $\begin{array}{l}\text { Alto } \\
\text { Alegre }\end{array}$ & 704 & Cana & Simples & IAC-OL3 & $25 / 10$ & 09/03 \\
\hline 37 & Getulina & 527 & Cana/Am. & Dupla & $\begin{array}{c}\text { Granolêic } \\
0\end{array}$ & $20 / 11$ & $16 / 03$ \\
\hline 38 & Borá & 483 & Cana & Dupla & $\begin{array}{c}\text { Granolêic } \\
0\end{array}$ & $17 / 11$ & 28/03 \\
\hline 39 & Varpa & 428 & $\begin{array}{c}\text { Pa./3xAm./Mand } \\
\text { ioca }\end{array}$ & Dupla & IAC-505 & $28 / 10$ & 28/03 \\
\hline 40 & Tupã & 404 & Cana & Simples & IAC-OL3 & $27 / 11$ & $01 / 04$ \\
\hline 41 & $\begin{array}{l}\text { Herculând } \\
\text { ia }\end{array}$ & 445 & Cana & Simples & IAC-OL3 & $26 / 11$ & $31 / 03$ \\
\hline 42 & lacri & 456 & Cana/Braquiária & Simples & $\begin{array}{c}\text { Granolêic } \\
0\end{array}$ & $01 / 12$ & $11 / 04$ \\
\hline 43 & $\begin{array}{l}\text { Martinópo } \\
\text { lis }\end{array}$ & 473 & Cana & Simples & IAC-505 & $28 / 11$ & $27 / 04$ \\
\hline 44 & Getulina & 435 & Cana/Am./Soja & Simples & $\begin{array}{c}\text { Granolêic } \\
0\end{array}$ & $20 / 11$ & 09/04 \\
\hline 45 & Getulina & 466 & Cana/Am.Mi & Dupla & $\begin{array}{c}\text { Granolêic } \\
0\end{array}$ & $13 / 11$ & --- \\
\hline
\end{tabular}




\section{Resultados e discussão}

Verifica-se nas "Figuras 1, 2 e 3" os resultados de produtividade de vagens das 45 áreas colhidas nas últimas três safras. Nota-se que é possível separar quatro faixas de produtividade (> 6200, 6200-5175, 5175$4125 \mathrm{e}<4125 \mathrm{~kg} \mathrm{ha}^{-1}$ ) variando a frequência em cada faixa, dependendo da chuva acumulada nos meses de dezembro e janeiro. A chuva e a produtividade média nas safras 2017/18, 2018/19 e 2019/20, foram respectivamente; $6.188 \mathrm{~kg} \mathrm{ha}^{-1}$ e $977 \mathrm{~mm}, 5.072 \mathrm{~kg} \mathrm{ha}^{-1}$ e $745 \mathrm{~mm}, 5.403 \mathrm{~kg}$ ha $^{-1}$ e $1016 \mathrm{~mm}$.

Levantamento microclimatológicos em 09 participantes comuns nos 02 anos, permite inferir que a deficiência hídrica na safra 2018/19 reduziu em $23 \%$ a produtividade média de amendoim na região. De acordo com pesquisas conduzidas na Índia, a deficiência reduziu entre 10 e $20 \%$ a produtividade do amendoim (NAAB et al., 2004). Verificou-se correlação positiva $(r=0,52)$ entre produtividade e chuva acumulada nos meses de verão (Tabela 4) somente nas duas primeiras safras. Na safra 2018/19, devido a ocorrência de veranico, o teor de matéria orgânica correlacionou-se melhor com a produtividade, do que com o teor de argila na cama de 0-40 $\mathrm{cm}$. Convém salientar nos três anos, semeaduras tardias penalizaram a produtividade, explicando $37 \%$ da diminuição na produtividade.

Com relação ao perfil técnico das lavouras, verificou-se que $60 \%$ dos cultivares eram do IAC (IAC-OL3, IAC-503 e IAC-505) e 40\% cv. Granolêico. A adoção de semeadoras com linha simples ou dupla, representou 49 e 51 $\%$, respectivamente. A reforma de canavial predominou com $47 \%$ das áreas, seguido por outros arranjos produtivos (milho, mandioca) com $31 \%$ e pastagem com $22 \%$. Esse é um fator importante, considerando que a maior frequência de altas produtividades foi observada em reforma de pastagem.

No que diz respeito à classificação pedológica dos solos das áreas avaliadas, não é possível apresentar resultados completos e conclusivo. Uma interpretação preliminar das duas primeiras safras, permite dizer que $64 \%$ das áreas tiveram os solos classificados com a legenda RQ-1 (argila < 
7\%) e RQ-2 (argila ente 8-15\%), que indicam ser da ordem NEOSSOLO Quartzarênico. As demais áreas foram identificadas, pela textura como $\mathrm{P}_{3} \mathrm{~A}$ (Argissolo latossólico, argila no $\mathrm{Hz} \mathrm{A}<15 \%$ e no $\mathrm{Hz} \mathrm{Bt}$ entre $16-25 \%$ ) com $29 \%$ das áreas, e somente uma classificada como L1(Latossolo textura média, argila 16-25\%). De acordo com Prado (2011), em termos de capacidade de armazenamento de água e potencial produtivo para cana-deaçúcar, as legendas seguem a seguinte ordem; $P_{3} A>L 1>R Q-2>R Q 1$. Contudo, no presente estudo as três maiores produtividades na safra 20171/8 foram nos produtores Flávio Colluci $\left(8.120 \mathrm{~kg} \mathrm{ha}^{-1}\right)$, Éder Tenório Bonfante $\left(7.521 \mathrm{~kg} \mathrm{ha}^{-1}\right)$ e Rodrigo Valochi $\left(7.035 \mathrm{~kg} \mathrm{ha}^{-1}\right)$, em solos classificados respectivamente com as legendas; $P_{3} A, P_{3} A$ e L1.

Para a safra 2018/19, os vencedores foram Cláudio Pimentel (7.056 $\mathrm{kg} \mathrm{ha}^{-1}$ ), Adilson Munhoz (6064 kg ha-1) e Aparecido Bidóia (6.012 kgha-1), em solos identificados, respectivamente, com as legendas RQ-1, RQ-2 e RQ-2, de acordo com a metodologia do IAC (PRADO et al., 2011).

Esses resultados sugerem que provavelmente a correlação entre produtividade de amendoim e unidade de classificação do solo, não seja a mesma que verificada para a cana-de-açúcar, pois existem outros fatores que determinam as altas produtividades. Adams et al. (1993) investigaram a relação entre o sistema de classificação de solos (nível de série e família), com a produtividade do amendoim, usando base de dados de 17 safras e 164 produtores.

Concluíram, que as produtividades foram significativamente afetadas pelo tipo de solo, mas também pelo ano (chuva), declividade e espessura do horizonte $\mathrm{A}$, no caso de solos com horizonte Bt.

Por outro lado, deve-se enfatizar que a textura do solo é reconhecida como um dos principais fatores que afetam o crescimento, desenvolvimento, produtividade e qualidade do amendoim. De acordo com Zhao et al. (2015), o número de flores e vagens por planta, bem como a biomassa das estruturas reprodutivas do amendoim são favorecidas por solos com baixos teores de argila na superfície. 


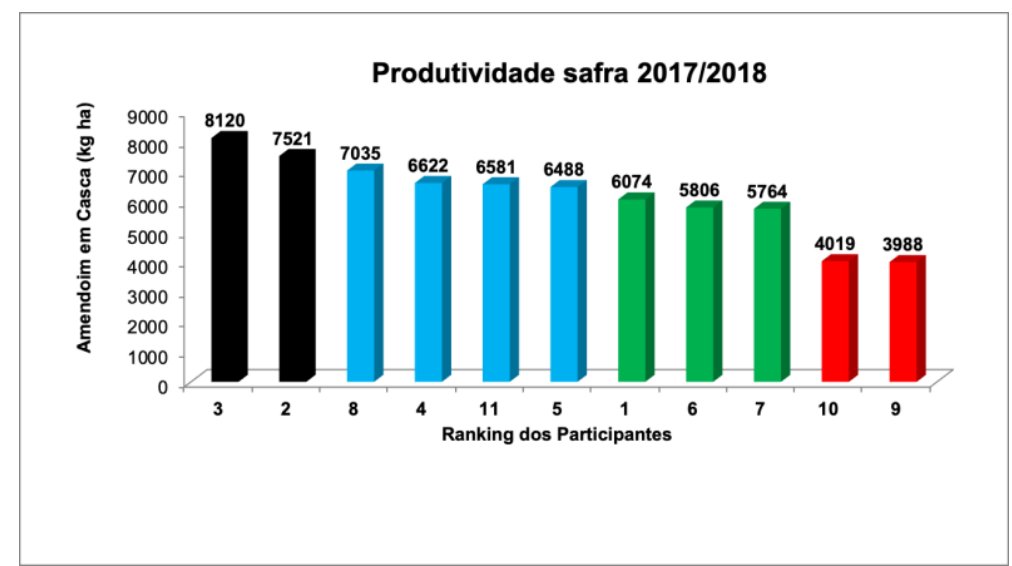

Figura 1. Distribuição das produtividades de amendoim ( $\mathrm{kg} \mathrm{ha}^{-1}$ ) na safra 2017/18. Região de Tupã/SP. Ranking segue numeração descrita na Tabela 1.

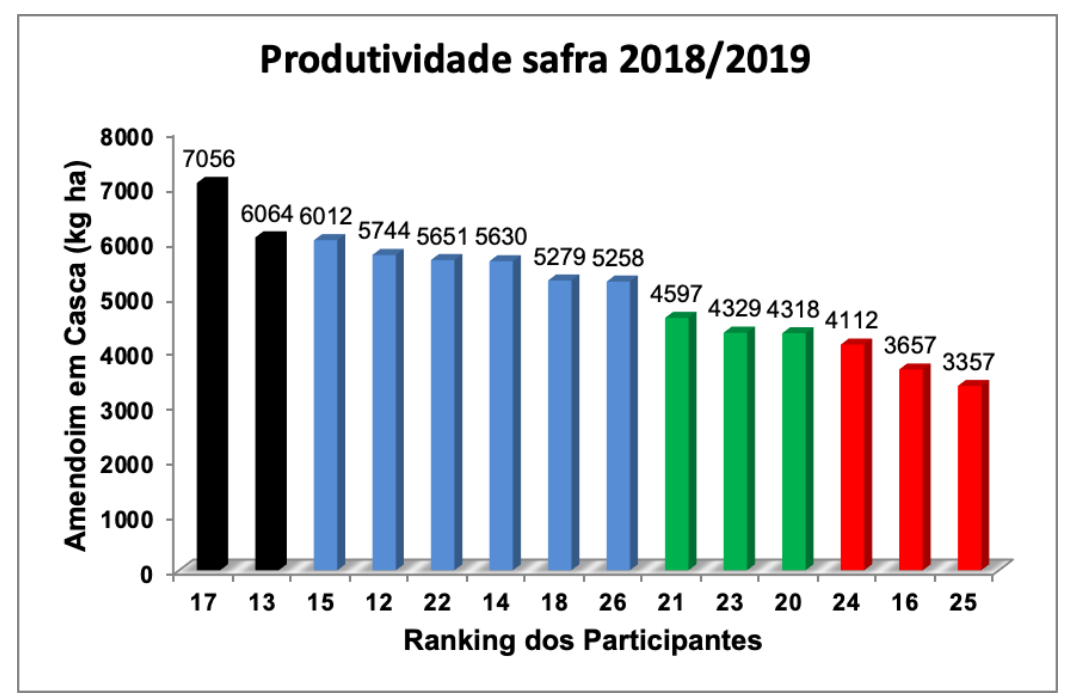

Figura 2. Distribuição das produtividades de amendoim $\left(\mathrm{kg} \mathrm{ha}^{-1}\right)$ na safra 2018/2019. Região de Tupã/SP. Ranking segue descrição da tabela 2.

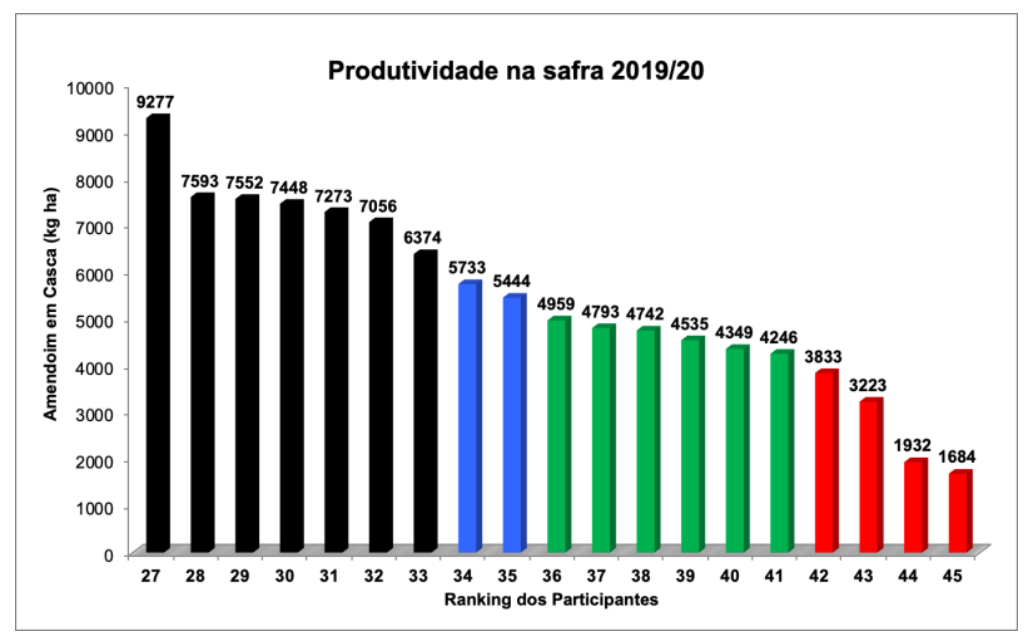

Figura 3. Distribuição das produtividades de amendoim $\left(\mathrm{kg} \mathrm{ha}^{-1}\right)$ na sa 2019/20. Região de Tupã/SP. Ranking segue descrição da Tabela 3. 
Tabela 4. Coeficientes de correlação simples ( $r$ ) entre a produtividade de amendoim em casca e algumas características do ambiente. Foram considerados 26 pares nas duas primeiras safras e 45 comparações nas três.

\begin{tabular}{|c|c|c|c|c|c|c|c|}
\hline Safras & Chuva & Chuva & Alt. & Época & Argila & $\mathrm{P}$ & M.O. \\
\hline 2018/2019 & $\mathrm{m}$ & $\mathrm{mm}$ & $\mathrm{m}$ & dias & $\%$ & $\mathrm{mg} \mathrm{dm}^{-3}$ & $\mathrm{~g} \mathrm{dm}^{-3}$ \\
\hline $\begin{array}{l}\text { Produtividade de } \\
\text { Amendoim }\end{array}$ & 0,38 & 0,52 & --- & --- & 0,14 & --- & 0,52 \\
\hline \multicolumn{8}{|l|}{ Três Safras } \\
\hline $\begin{array}{l}\text { Produtividade de } \\
\text { Amendoim }\end{array}$ & 0,10 & 0,11 & $\begin{array}{c}0,2 \\
4\end{array}$ & $-0,37$ & --- & 0,38 & --- \\
\hline
\end{tabular}

\section{Conclusões}

Foram identificadas 4 faixas de produtividades; > 6200, 6200-5175, $5175-4125 \mathrm{e}<4125 \mathrm{~kg} \mathrm{ha}^{-1}$, com frequência em cada faixa variando conforme a ocorrência de veranicos, o qual chegou a impactar em $23 \%$ o rendimento na safra $2018 / 19$. Altos tetos produtivos (> $7200 \mathrm{~kg} \mathrm{ha}^{-1}$ ) foram verificados em solos com baixos teores de argila $(0-40 \mathrm{~cm})$, indicando que os fatores chuva acumulada, teores de matéria orgânica e histórico são determinantes na caracterização de ambientes de produção para o amendoim.

\section{Agradecimentos}

Os autores agradecem ao CNPq pelas bolsas de produtividade DT2, bem como aos produtores participantes nessas três safras, ao apoio da empresa TIMAC Agro no custeio das avaliações, ao suporte logístico da empresa AGROTEKENE. Ao Prof. Dr. Paulo Cesar Sentelhas (ESALQ/USP) pelo fornecimento dos dados climatológicos (2017/18 e 2018/19) viabilizados pelas StartUp Agrymet e Agrymax. Ao LabSolo da FEAGRI/UNICAMP pelas análises de textura das amostras de solo e ao Dr. Hélio do Prado pela ajuda na identificação de algumas legendas de classificação dos solos. 


\section{Referências}

ADAMS, J.F.; HAJEK, B.F.; DAVIS, D.P.; HARTZOG, D.L. Relationship between peanut yield and soil classification of Ultisoils in southeastern Alabama. Agricultura, Ecosystems and Environment, v. 43, p. 79-86, 1993.

CONAB - Companhia Nacional de Abastecimento. Acompanhamento da Safra Brasileira - Séries históricas, amendoim safra total, julho 2020. Disponível em: <https://www.conab.gov.br/info-agro/safras/graos>.

LANDELL, M.G.A.; PRADO, H.; VASCONCELOS, A.C.; PERECIN, D.; ROSSETO, R.; BIDÓIA. M.A.P.; SILVA, M.A.; XAVIER, M.A. Oxisol subsurface related to sugarcane productivity. Scientia Agrícola, v. 60, n.4., p. 741-745, 2003.

NAAB, J.B.; SINGH, P.S.; BOOTE, K.J.; JONES, J.W.; MARFO. K.O. Using the CROPGRO-Peanut model to quantify yield gaps of peanut in the Guinea Savana of Ghana. Agronomy Journal, v, 96, n. 5., p. 1231-1242, 2004.

PRADO, H. Pedologia Fácil - Aplicações. $3^{a}$ Edição- Revista e Ampliada. 2011, $180 \mathrm{p}$.

SENTELHAS, P.C.; BATTISTI, R.; CÂMARA, G.M.S.; FARIAS, J.R.B.; HAMPF, A.; NENDEL, C. The soybean yield gap in brazil magnitude, causes and possible solutions for a sustainable production. Journal of Agricultural Science, v. 153, p. 1394-1411, 2015.

SONAWANE, K.G.; PORKHARKAR, V.G.; GULAVE, C.M. Impact of improved production technology of groundnut (Arachi hypogaea L.) on farm productivity and income in Western Maharashtra. J. Oilseeds Res., v. 33, n. 2, p. 138-145, 2016.

ZHAO, C.X.; JIA, L.H.; WANG, Y.F.; WANG, M.L.; McGIFFEN Jr., E. Effect of diferente soil texture on peanut growth and development. Communications in Soil Science and Plant Analysis, v. 46, p. 22492257, 2015. 